\title{
Modeling spatiotemporal dynamics of outbreaking species: influence of environment and migration in a locust
}

\author{
Sophie Veran, ${ }^{1}$ Stephen J. Simpson, ${ }^{2}$ Gregory A. Sword, ${ }^{3}$ Edward Deveson, ${ }^{4,5}$ Sylvain Piry, ${ }^{1}$ James E. Hines, ${ }^{6}$ \\ AND KARINE BERThIER $2,7,8$ \\ ${ }^{1}$ INRA, UMR CBGP, F-34988 Montferrier-sur-Lez Cedex, France \\ ${ }^{2}$ School of Biological Sciences, University of Sydney, New South Wales 2006 Australia \\ ${ }^{3}$ Department of Entomology, Texas A\&M University, College Station, Texas 77843-2475 USA \\ ${ }^{4}$ Australian Plague Locust Commission, Australian Government Department of Agriculture, Fisheries and Forestry, GPO Box 858 , \\ Canberra, Australian Capitol Territory 2601 Australia \\ ${ }^{5}$ Fenner School of Environment and Society, Australian National University, Canberra 0200 Australia \\ ${ }^{6}$ USGS Patuxent Wildlife Research Center, Laurel, Maryland 20708 USA \\ ${ }^{7}$ INRA, UR0407 Pathologie Végétale, F-84143 Montfavet, France
}

\begin{abstract}
Many pest species exhibit huge fluctuations in population abundance. Understanding their large-scale and long-term dynamics is necessary to develop effective control and management strategies. Occupancy models represent a promising approach to unravel interactions between environmental factors and spatiotemporal dynamics of outbreaking populations. Here, we investigated population dynamics of the Australian plague locust, Chortoicetes terminifera, using density data collected between 1988 and 2010 by the Australian Plague Locust Commission over more than 3 million $\mathrm{km}^{2}$ in eastern Australia. We applied multistate and autologistic multi-season occupancy models to test competing hypotheses about environmental and demographic processes affecting the large-scale dynamics of the Australian plague locust. We found that rainfall and land cover predictors best explained the spatial variability in outbreak probability across eastern Australia. Outbreaks are more likely to occur in temperate than tropical regions, with a faster and more continuous response to rainfall in desert than in agricultural areas. Our results also support the hypothesis that migration tends to propagate outbreaks only locally (over distances lower than $400 \mathrm{~km}$ ) rather than across climatic regions. Our study suggests that locust outbreak forecasting and management systems could be improved by implementing key environmental factors and migration in hierarchical spatial models. Finally, our modeling framework can be seen as a step towards bridging the gap between mechanistic and more phenomenological models in the spatial analysis of fluctuating populations.
\end{abstract}

Key words: Chortoicetes terminifera; climate; cluster; dispersal; landscape; locust; multistate; occupancy; outbreak; pest species; spatial autocorrelation.

\section{INTRODUCTION}

A feature of numerous pest species is extreme fluctuations in population density, leading to dramatic outbreaks with consequent agricultural and economic impacts over large areas (e.g., rodents [Singleton et al. 2010], locusts [Pener and Simpson 2009], and moths [Baltensweiler and Fischlin 1988]). Understanding their large-scale and long-term dynamics, and more specifically how outbreak patterns are shaped by the interplay between the spatiotemporal variability of environmental factors (e.g., climate) and demographic processes (e.g., dispersal), is necessary to develop more effective control and management strategies and to predict outbreak risks

Manuscript received 28 January 2014; revised 8 July 2014; accepted 7 August 2014. Corresponding Editor: J. D. Reeve.

${ }^{8}$ Corresponding author. Karine Berthier INRA, UR0407 Pathologie Végétale, F-84143 Montfavet, France.

E-mail: karine.berthier@avignon.inra.fr due to global warming and land use change (Knape and de Valpine 2011).

The analysis of time series count data is an important tool for studying fluctuating populations (see for examples Bjørnstad et al. 1999, Liebhold et al. 2004, Goswami et al. 2011, Knape and de Valpine 2011). However, demographic time series remain scarce for most species and many data sets that have proven to be critical for studying long-term population dynamics were not collected for research purposes, but rather derived from historical reports of occurrence, hunting statistics, or pest management monitoring (e.g., lynx [Stenseth et al. 1998], locust [Stige et al. 2007, Tian et al. 2011], moth [Johnson et al. 2004, 2006]). One of the main challenges when using these data is that abundance information is often not systematically recorded from the same locations and at regular time intervals, resulting in time series plagued with missing data. Studies based on such data may need to either pool spatial information to address the long-term temporal 
component of population dynamics (e.g., Stige et al. 2007, Tian et al. 2011), or exclude temporal or spatial samples with insufficient information (e.g., Kvasnes et al. 2010). Moreover, methods such as auto-regressive models fitted to time series data are phenomenological (or correlative) statistical models. Population dynamics result from a complex interaction between linear and nonlinear (such as density-dependent) processes acting at different spatial and temporal scales. Phenomenological models are, therefore, limited in their ability to explain current population patterns causally and predict the future state of biological systems. Achieving these goals requires the development of more mechanistic models (Bjørnstad and Grenfell 2001, Benton et al. 2006).

In this context, multistate, multi-season, occupancy models provide a promising approach to unraveling interactions between environmental factors and spatiotemporal dynamics of fluctuating populations (Nichols et al. 2007). Site occupancy models were first developed to estimate species occurrence while accounting for errors in species detection (MacKenzie et al. 2002). The recent extension of these models to deal with multiple states considerably increases their applicability. They can now be used to consider multiple biologically relevant states such as multiple classes of abundance or breeding/nonbreeding states (MacKenzie et al. 2009, Martin et al. 2009). Moreover, occupancy models can also incorporate spatial autocorrelation, which occurs when the values of response variables sampled at nearby locations are not independent from each other (Bled et al. 2010, Yackulic et al. 2012). Spatial autocorrelation can be quantified using geostatistics such as Moran's correlograms, spline correlograms, or variograms, and can be explicitly included in models of spatial distribution using an autologistic component (Rossi et al. 1992, Liebhold et al. 1993, Augustin et al. 1996, Bjørnstad et al. 1999, Dormann et al. 2007). Adding an autologistic component to occupancy models allows for the testing of a contagious-type process hypothesis, such as the expansion of invasive species (Bled et al. 2010, Yackulic et al. 2012). Lastly, occupancy models account for imperfect detection, specifically the inability of investigators to determine species' absence (false negative; MacKenzie et al. 2003) and more recently species' presence (false positive) at a sample site (Royle and Link 2006, Miller et al. 2011). Failing to account for imperfect detection can lead to a discrepancy between observation data and the true state of the system, and can therefore produce biased estimates of transition probabilities (MacKenzie 2006, Veran et al. 2012).

An interesting biological model for applying occupancy models is provided by locust populations, which are one of the most striking and notorious examples of populations exhibiting huge fluctuations in density. Locust outbreaks and their subsequent mass migrations are preceded by the expression of a densitydependent phenotypic phase change, from the relative- ly harmless solitarious phase at low densities to the gregarious phase at high densities. The resulting formation of dense bands of marching juveniles and flying adult swarms are responsible for severe agricultural and economic losses across very large areas on multiple continents (Simpson et al. 1999, Gray et al. 2009, Pener and Simpson 2009). In this study, we focus on the Australian plague locust (APL), Chortoicetes terminifera, for which 23 years of survey data have been recorded across more than 3 million $\mathrm{km}^{2}$ in eastern Australia as part of a rapid index-monitoring program developed by the Australian Plague Locust Commission (APLC) for locust outbreak forecasting and management.

Emergence of locust outbreaks is known to be primarily dependent on rainfall events and high temperatures (optimum for egg development around $30-35^{\circ} \mathrm{C}$ ), which locally provide suitable habitat conditions for rapid population growth, namely abundant herbaceous vegetation for food and soil moisture for egg laying and embryological development (Popov et al. 1991, Hunter et al. 2001, Sword et al. 2010, Deveson 2013). Local patterns of resource abundance, quality, and distribution determine whether solitarious populations will become aggregated and undergo the transition to a migratory gregarious phase (Babah and Sword 2004, Despland et al. 2004). Considering the diversity of climate and habitats across eastern Australia, both the quality and amount of resources for locust population growth are likely to vary strongly in space and time. Indeed, the southern part of eastern Australia is climatically temperate with a uniform winter rainfall distribution, whereas the northern part is subject to strong tropical influences with heavier rains in summer (Bryant 1985, Hendon et al. 2007). Eastern Australia is also highly variable in terms of land cover. The western part is mainly an arid desert with very ephemeral and scattered vegetation patches, while the central portion corresponds to the agricultural belt from north to south. This spatial variation in food resources may locally limit several factors affecting the spatiotemporal dynamics of locust populations including the intrinsic rate of population increase, the maintenance of high-density populations over several successive generations, and the ability of migrating individuals to find favorable habitats. APL adults have the ability to fly distances ranging between 200 and $500 \mathrm{~km}$ overnight (Drake and Farrow 1983, Farrow 1990, Bryceson 1991). Wright (1986) even suggested that gregarious movements could occur over distances up to $1500 \mathrm{~km}$ in three or four nights. The record of southward long distance movements, and of dramatic late summer and autumn outbreaks in agricultural areas of southeastern Australia have long supported the idea that the northern central arid area of Australia was the main source of outbreaks, from which locusts migrated en masse over two to three successive generations in a year (Symmons and Wright 
A
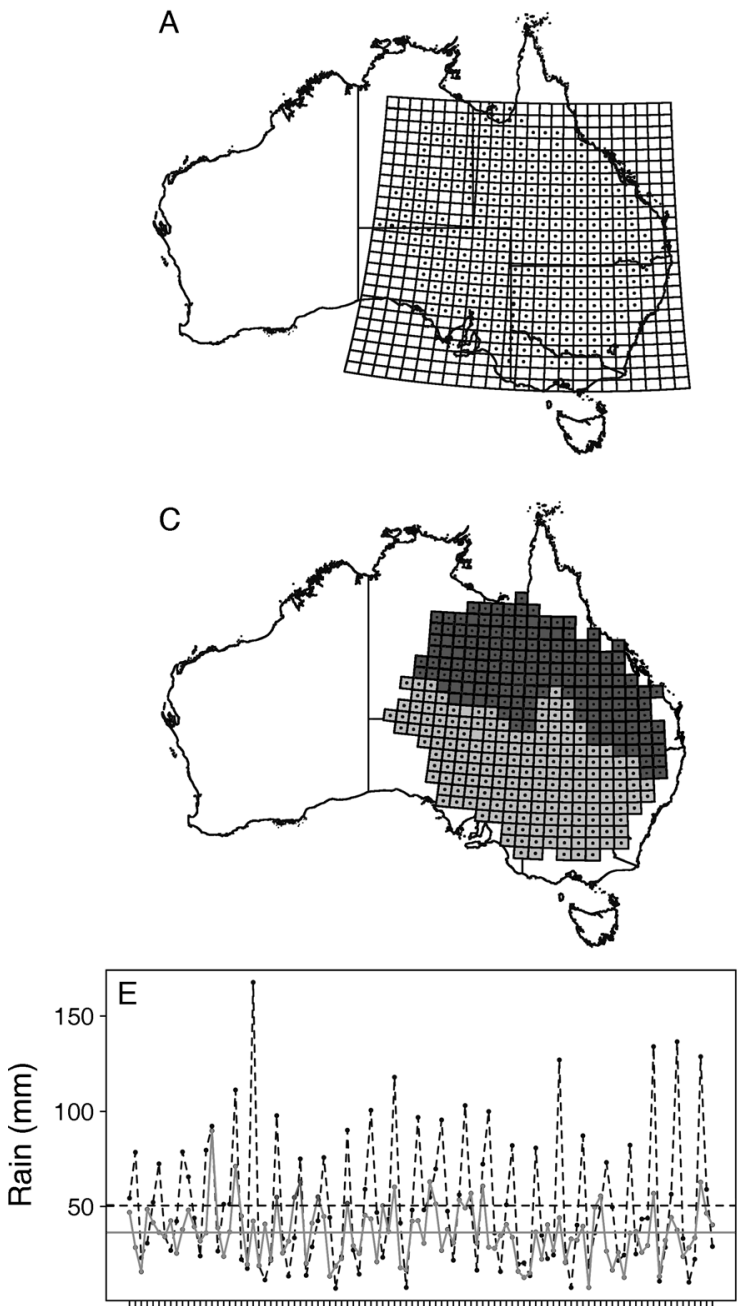

Time (season)

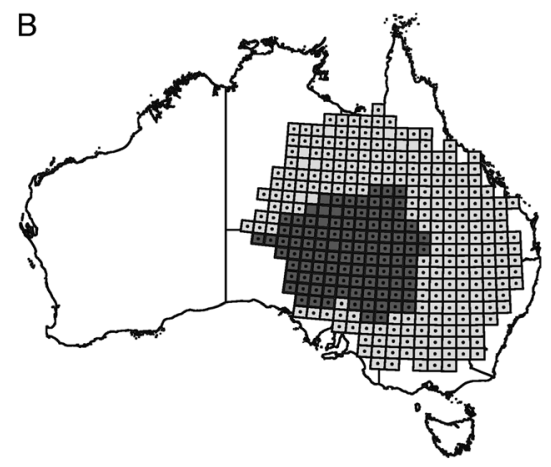

$\mathrm{D}$
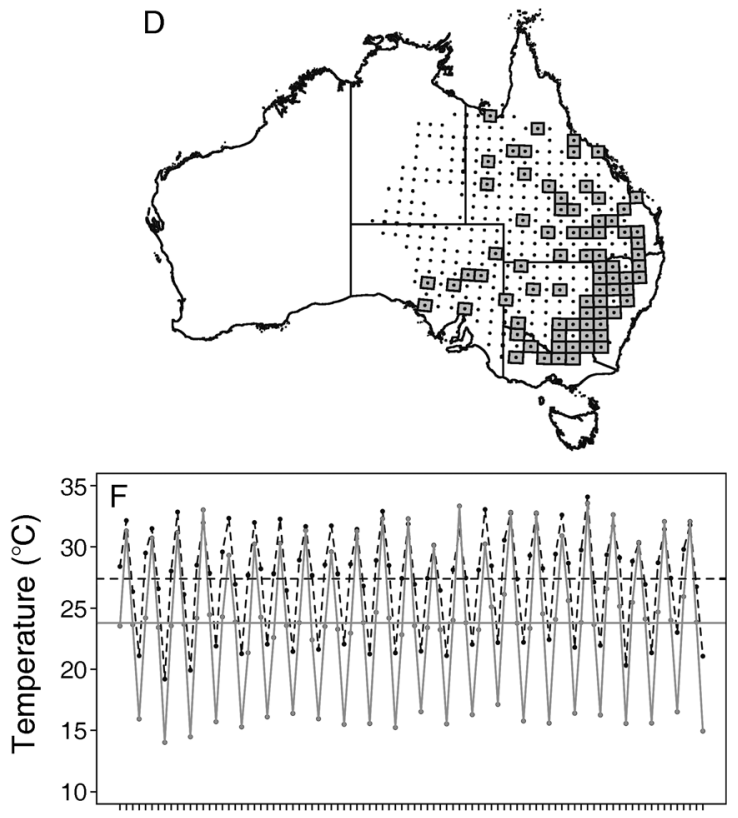

Time (season)

FIG. 1. The study area. (A) The $100 \times 100 \mathrm{~km}$ grid cells showing the localization of locust records (black dots). (B) The landscape typology: grassland (light gray) and desert (dark gray). (C) The result of the clustering analysis on the 23-year rainfall time series that divides Eastern Australia into a northern tropical region (dark gray) and a southern temperate region (light gray). (D) The localization of the cells for which temperature data were available. The seasonal time series of (E) rainfall and (F) temperature from the northern (black dotted lines) and southern (gray solid lines) clusters. Annual data from the seasons spring (September, October, and November), summer (December, January, and February), autumn (March, April, and May) and winter (June, July, and August) were averaged over all cells from each rainfall-based cluster. Horizontal lines in (E) and (F) show the mean values for rainfall and temperature calculated over the 23 years of data within the northern (dotted lines) and southern (solid lines) clusters.

1981, Bryceson and Wright 1986, Wright 1987, Wright and Symmons 1987).

Although both environmental features (rainfall, temperature, and vegetation cover) and migration are clearly identified as key factors in locust outbreaks, little attempt has actually been made to assess their relative contribution in the spatiotemporal dynamics of locust populations (Deveson et al. 2005). Here, we applied multistate and autologistic multi-season occupancy models to investigate how the interplay between environmental heterogeneity and migration drive the large-scale outbreak dynamics of APL populations.

\section{Methods \\ Locust density data}

Densities of locust nymphs were recorded each month from 1988 to 2010 by the APLC at numerous sites across eastern Australia, mostly in Queensland and New South Wales (Fig. 1A; details available online). ${ }^{9}$ At each site, located at least $10 \mathrm{~km}$ apart, a measure of nymph density was recorded along a 250-m transect. Nymph density was then transformed into a density scale with the following indices: 0 , absence of nymphs; 1 , scattered

${ }^{9}$ http://www.daff.gov.au/animal-plant-health/locusts 
populations of nymphs (density $\leq 30 / \mathrm{m}^{2}$ ); and 2 , high density of gregarious nymphs (density $>30 / \mathrm{m}^{2}$ ). The threshold of 30 nymphs $/ \mathrm{m}^{2}$ was chosen because APLC field experience indicated a high probability of detection and gregarious behavior above that density. The density index of 2 is hereafter referred to as "outbreaking" populations, as it generally refers to densities of hundreds to thousands of nymphs per square meter forming large bands exhibiting coordinated movements.

To study the spatiotemporal dynamics of locust outbreaks, we divided the survey area into a regular grid with a cell size of $100 \times 100 \mathrm{~km}$ (Fig. 1A). This resolution allowed discrimination of the spatial variation in key environmental factors (i.e., southern temperate vs. northern tropical regime and western desert vs. eastern agricultural areas) and migration (i.e., occurring over hundreds of kilometers) without excessively decreasing within-cell data availability. All monthly density indices collected between 1988 and 2010 were attributed to a specific grid cell and season. Seasons consistent with the multivoltine life cycle of the APL were defined as follows: data collected in September, October, and November were attributed to the first season (Spring); data collected in December, January, and February were attributed to the second season (Summer); and data collected in March, April, and May were attributed to the third season (Autumn). In June, July, and August (Winter), nymphs are generally not present, but a high proportion of eggs laid in autumn enter diapause and only resume development in late winter (Wardhaugh 1986). Thus, we did not include the winter season in the analysis and considered the system to resume from its last state at the following season. The final data set consisted of 323 cells at 70 occasions (i.e., 23 years $\times 3$ seasons per year, and a first season to initiate the model). When several measures of density existed in the same cell at a given season, they were considered as survey replicates in our analysis. The number of survey replicates per cell and per season varied between 1 and 15, with an average between 1.29 and 2.98 replicates per cell per occasion.

\section{Environmental covariates}

Rainfall and temperature.-We retrieved monthly rainfall, and temperature maxima and minima measures, recorded between 1988 and 2010, from many stations of the Australian Bureau of Meteorology scattered across eastern Australia (data available online). ${ }^{10}$ As the temperature values were highly correlated $(>90 \%)$, we only used maximum temperature in the analyses. When several meteorological stations belonged to the same grid cell we averaged the monthly rainfall and temperature values retrieved from each station and then over each season in order to obtain a single climatic times series for each cell of the grid. We performed hierarchi- cal clustering analyses on seasonal rainfall and temperature time series using Ward's minimum variance method (Ward 1963). The clustering analysis that was based on rainfall data clearly segregated two regions of eastern Australia along a North-South axis, with a northern region exposed to heavier rains and warmer temperatures (i.e., tropical climate) and a southern temperate climate (Fig. 1C, E, F). Analysis of the maximum temperature data produced a similar pattern supporting the binary regionalization based on rainfall data. However, due to the lack of temperature data for many cells (Fig. 1D), we only used the result of the cluster analysis conducted on rainfall as a spatial categorical covariate, assigning each cell to a category of either northern cluster or southern cluster as a proxy of tropical vs. temperate climate.

Vegetation cover.-To investigate the impact of spatial variation in vegetation cover on the dynamics of outbreaks, we defined a landscape typology based on the proportion of herbaceous vegetation that was taken as a proxy of food resources for locusts. Our land cover typology was based on a vegetation continuous field (VCF), a raster image of 250-m resolution derived from all seven bands of the Moderate-resolution Imaging Spectroradiometer (MODIS) sensor onboard NASA's Terra satellite (data available online). ${ }^{11}$ We used the proportional estimates for woody vegetation, herbaceous vegetation, and bare ground provided by the VCF image to classify each $100 \times 100 \mathrm{~km}$ cell to one of these categories based on the highest proportion of vegetation cover type within each cell. This classification clearly segregated the study area into two land covers: herbaceous vegetation and bare ground, which we will hereafter consider as representative of grassland and desert areas (Fig. 1B).

\section{Multistate occupancy modeling}

A key aspect of accounting for the spatial and temporal dynamics of locust outbreaks is distinguishing between the impacts of environmental factors vs. migration. Indeed, transitions from solitarious to gregarious populations could arise from either local intrinsic growth due to favorable environmental conditions or through migration from other gregarious populations. In the former case, we would expect such a transition from low density to gregarious populations to be correlated with environmental covariates such as rainfall or vegetation cover, whereas under the hypothesis of migration, a transition from absence or low density to high density should strongly depend on the presence of gregarious populations in the neighborhood of the site considered. Therefore, testing for the alternative hypotheses requires modeling and estimating the different transitions: from low density to gregarious populations and from the absence of locusts to 
TABle 1. Covariates used for modeling the probabilities of transitions $\psi^{1,2}$ and $\psi^{0,2}$ and the probability of misclassification $p^{0,1}$.

\begin{tabular}{llc}
\hline \hline Abbreviation & & \multicolumn{1}{c}{ Covariate description } \\
\hline LatLong & latitude and longitude (quadratic) & Level of variation \\
LC & land cover (desert vs. grassland) & space \\
Clu & regions (north vs. south) resulting from the cluster analysis of weather variables & space \\
$R$ & amount of rainfall per season in mm (quadratic) & space \\
$T$ & temperature per season in ${ }^{\circ} \mathrm{C}$ (quadratic) & space and time \\
$S$ & seasonal effect & space and time \\
$y$ & annual effect & time \\
$O$ & occasion (variation for each season and each year) & time \\
$\hat{\pi}_{\text {local }}$ & local neighborhood effect (distance up to 400 km from the focal cell) & time \\
$\hat{\pi}_{\text {regional }}$ & regional neighborhood effect (all cells within the study area) & space and time \\
$i$ & constant & space and time \\
\hline
\end{tabular}

Notes: All covariates were standardized to have a mean of 0 and a standard deviation of 1 except $\hat{\pi}_{\text {local }}$, which that ranges between 0 and 1 , and LC and Clu, which are both discrete covariates. The three states of the system are sites with no locust present $(0)$, sites with a low population density (1), and sites with a gregarious population (2). The variable $\psi^{R, S}$ is the probability that a site in state $R$ at time $t$ will be in state $S$ at time $t+1$.

gregarious populations (all covariates used for modeling the probabilities of transitions are presented in Table 1).

As errors of classification are inherent when collecting data, there is a risk of a discrepancy between the observed abundances and the true abundances, and therefore a risk of biased estimates of the different rates of transition (MacKenzie 2006, Veran et al. 2012). In our case, misclassifying gregarious populations is very unlikely since locust detection is straightforward as densities reach hundreds to thousands of nymphs per square meter. Detecting scattered populations is often more problematic and, in such a situation, failing to account for a low rate of detection may lead to falsely classifying a site as having no locusts despite scattered populations being present. This could create biases in estimated transitions (especially overestimating the transition from sites free of locusts to gregarious populations), and in estimated regression coefficients (reflecting associations between transitions and the different factors [MacKenzie 2006]), that could lead to erroneous conclusions.

In this study, we applied the multistate site occupancy model described by MacKenzie et al. (2009) to the locust data set to estimate the probabilities of transition between different categories of abundance (or states) while accounting for errors of misclassification. A site (a cell in this study) could be in any of the following three different states $(R)$ : unoccupied $(R=0)$, occupied with a low-density population $(R=1)$, and occupied with a gregarious population $(R=2)$. We define $\psi^{R, S}$ as the probability that a cell in state $R$ at time $t$ will be in state $S$ at time $t+1$. For example, $\psi_{t}^{0,2}$ and $\psi_{t}^{1,2}$ would describe an outbreak event: the transition from a cell without locusts (state 0 ) or with a low-density population (state 1) at time $t$ to a site with a gregarious population (state 2) at time $t+1$ (Fig. 2). It is important to note that the number of transitions increases with the square of the number of states. A trade-off is necessary between a high number of states to accurately describe the biological system and maintaining a low number of states to be able to estimate all transitions from the data set.

To test for the potential impact of environmental factors on the probability of transition $\psi^{1,2}$, the transition from a cell with low density of locust to an outbreak, we modeled the probability of $\psi^{1,2}$ as a function of the different predictors $\mathbf{Y}_{t}$ to estimate the $\beta$ coefficients using a multinomial-logit function where the transformed $\psi_{t}^{1,2}$ is equal to $\beta \times \mathbf{Y}_{t}$. Hence the backtransformed parameter is

$$
\psi_{t}^{1,2}=\frac{\exp \left(\mathbf{Y}_{t} \beta\right)}{1+\exp \left(\mathbf{Y}_{t} \beta\right)+\delta_{t}^{1,1}}
$$

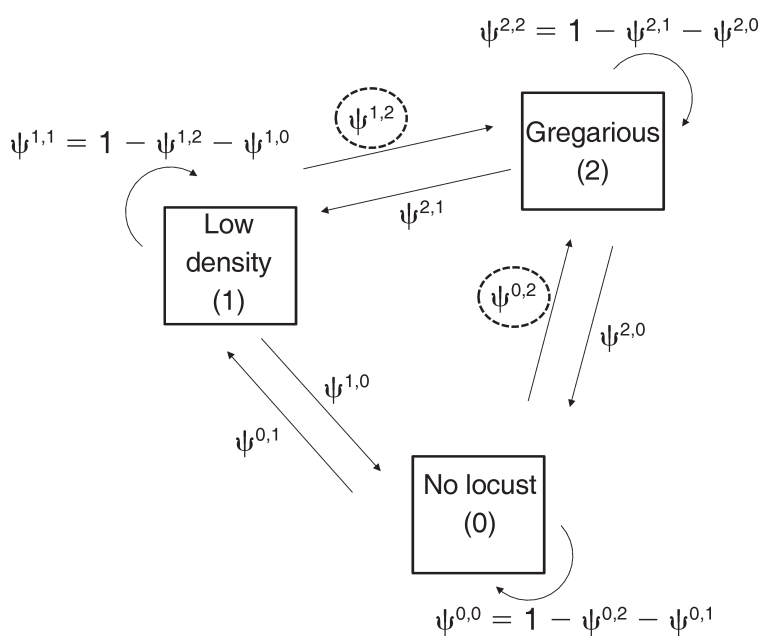

FIG. 2. Diagram illustrating the possible state transitions and associated parameters. The three states of the system are sites with no locust present (0), sites with a low population density (1), and sites with a gregarious population (2). The variable $\psi^{R, S}$ is the probability that a site in state $R$ at time $t$ will be in state $S$ at time $t+1$. Probabilities of outbreaks (denoted by dashed circles) are the probabilities of having a gregarious population at time $t+1$ when the site had no locusts or a low population density at the previous time step $\left(\psi^{0,2}\right.$ and $\left.\psi^{1,2}\right)$. Note that since the sum of all transitions from one state to any other state equals one, any of these transitions can be estimated indirectly from the others (for example $\psi^{0,0}=1-\psi^{0,2}-\psi^{0,1}$ ). 
where $\delta_{t}^{1,1}$ is the transformed parameter $\psi_{t}^{1,1}$. Such a multinomial-logit function guarantees the sum of transitions from one state to any other to be less than or equal to one; as a consequence, back-transformed parameters are no longer independent. In order to maintain the relationship between $\psi_{t}^{1,2}$ and environmental factors, all other transitions were assumed to be constant, except two redundant parameters $\psi^{1,0}$ equals to $1-\psi^{1,2}-\psi^{1,1}$ and $\psi^{0,1}$ equals to $1-\psi^{0,2}-\psi^{0,0}$.

Data required for inference about $\psi_{t}^{R, S}$ are the state histories associated with each grid cell in the area of interest. For example, the history: 11012 represents a cell, assessed on five different occasions, where a lowdensity population of nymphs had been detected during the first two occasions, no nymphs detected at the third, a low-density population detected at the fourth occasion, and a gregarious population at the fifth. Assuming no errors in state classification and constant transition probabilities over time, we could model this history as $\operatorname{Pr}\left(\begin{array}{lllll}1 & 1 & 0 & 1 & 2\end{array}\right)=\pi^{1} \psi^{1,1} \psi^{1,0} \psi^{0,1} \psi^{1,2}$, where $\pi^{R}$ is the probability that a randomly selected grid cell at time $t$ is in state $R$.

The above expression represents the probability that a randomly selected cell at some initial time $t$ shows this sequence of densities. Using the same reasoning for each cell over all occasions, we can estimate the initial state and transition probability parameters with a maximum likelihood approach.

To account for state misclassification, we used the different measures of density assigned to the same cell at a given time as different replicates. Let $p_{j}^{l, m}$ be the probability of observing a cell in state $l$ during the survey $j$ given the true occupancy state is $m$. In the general framework of multistate models, potential misclassification of units from the observed data extends in one direction (MacKenzie et al. 2009). That is, if the species is not detected during a survey, it could be in any of the three states; if observed in state 1 , it can be in either states 1 or 2 ; and, if observed in state 2 , it is truly in state 2 . We added constraints on the potential misclassification of states by assuming that the species cannot be misclassified when observed as gregarious $\left(p_{j}^{0,2}=0\right.$ and $\left.p_{j}^{1,2}=0\right)$

$$
\begin{array}{ll} 
& \multicolumn{4}{c}{\text { Observed state }} \\
0 & 0 \\
\text { True state } & 1 \\
& 2
\end{array}\left[\begin{array}{ccc}
1 & 0 & 0 \\
1-p_{j}^{1,1} & p_{j}^{1,1} & 0 \\
0 & 0 & 1
\end{array}\right] .
$$

To comply with this assumption, when a gregarious population was detected at one occasion in a cell, we removed all other existing survey data from that same occasion. We assumed that if other states had been observed in the same cell, it was due to spatial or temporal heterogeneity (surveys conducted earlier or later than those where the locusts were observed as gregarious), but the state of the cell during this occasion was unambiguously that of having a gregarious population. Outbreaking populations of gregarious locusts reach very high densities of hundreds to thousands of nymphs per square meter, which form huge bands ranging from hundreds of meters to hundreds of kilometers, and exhibit coordinated movements that can in no way be confounded with scattered populations of solitarious locusts. Moreover, in addition to the surveys conducted on the ground, the APLC routinely uses aerial surveys from planes to monitor large areas for high-density populations because migratory bands can be spotted from the air. They also receive alerts from landholders (who are sensitive to the risk of outbreaks and associated financial impacts) when they spot conspicuous groups of locusts on their property or moving across roads and highways. Thus, for a given season (three consecutive months) and site, the presence of small outbreaking populations is likely to be detected with much more accuracy than that of low-density populations.

Finally, if a cell is not surveyed at a time $t$, it provides no information to the estimation of the transition probabilities between time $t-1$ and $t$ and time $t$ and $t+1$.

\section{Autologistic modeling}

Autologistic models have proven to be useful for modeling range expansion of invasive species by incorporating the effect of neighborhood occupancy on the state of a given location (Bled et al. 2010, Yackulic et al. 2012). There is a conceptual analogy between range expansion of invasive species and mass migration in pests; the likelihood of a site that is free of invasive species becoming colonized is expected to increase if the surrounding area is already colonized. In the same way, a site is more likely to have a gregarious population if sites surrounding it have gregarious populations.

The average probability of a site having a gregarious population within a neighborhood of $n_{i}$ sites can be estimated by

$$
\hat{\bar{\pi}}_{t}^{n_{i}}=\frac{1}{l_{i}} \sum_{j \in\left\{n_{i}\right\}} \pi_{j, t}
$$

where $l_{i}$ is the number of sites located in the neighborhood of focal site $i$, and $\pi_{j, t}$ is the estimated probability that a neighboring site $j$ has a gregarious population at time $t$ (Yackulic et al. 2012). Neighborhoods can be defined in different ways (Bled et al. 2010, Yackulic et al. 2012). In this study, we compared hypotheses about migrating distance covered by gregarious populations, which lead to outbreak diffusion (Deveson et al. 2005, Chapuis et al. 2011). Under the hypothesis of local migration, gregarious individuals will move only to areas that are close to their emergence site. We then defined a local neighborhood as the cells located between 100 and $400 \mathrm{~km}$ from the focal cell (i.e., a square of $7 \times 7$ cells centered on the focal cell). Such a distance falls in the range of the maximum empirical estimates of locust overnight flight capacities, i.e., 200-500 km (Drake and Farrow 1983, Bryceson and Wright 1986, Farrow 1990). 
TABle 2. Multistate model selection results for the Australian locust data set.

\begin{tabular}{|c|c|c|c|c|c|c|}
\hline$\psi^{0,2}$ & $\psi^{1,2}$ & $p^{0,1}$ & $\triangle \mathrm{AIC}$ & $w$ & $k$ & Deviance \\
\hline$i$ & $\mathrm{Clu} \times \mathrm{LC} \times R+T$ & $y$ & 0 & 0.817 & 42 & 14666 \\
\hline$i$ & $\mathrm{Clu} \times \mathrm{LC} \times R$ & $y$ & 3 & 0.182 & 40 & 14673 \\
\hline$i$ & $\mathrm{Clu} \times \mathrm{LC}+R$ & $y$ & 17 & $<0.001$ & 34 & 14699 \\
\hline$i$ & LatLong $\times S$ & $y$ & 19 & $<0.001$ & 43 & 14683 \\
\hline$i$ & LatLong & $y$ & 32 & $<0.001$ & 33 & 14716 \\
\hline$i$ & $\mathrm{Clu} \times \mathrm{LC} \times S$ & $y$ & 64 & $<0.001$ & 40 & 14734 \\
\hline$i$ & $\mathrm{LC} \times R$ & $y$ & 88 & $<0.001$ & 34 & 14770 \\
\hline$i$ & $\mathrm{Clu} \times \mathrm{LC}$ & $y$ & 100 & $<0.001$ & 32 & 14786 \\
\hline$i$ & $R$ & $y$ & 107 & $<0.001$ & 31 & 14800 \\
\hline$i$ & $\mathrm{Clu}$ & $y$ & 128 & $<0.001$ & 30 & 14824 \\
\hline$i$ & $\mathrm{LC}$ & $y$ & 154 & $<0.001$ & 30 & 14844 \\
\hline$i$ & $i$ & $y$ & 318 & $<0.001$ & 28 & 15012 \\
\hline$i$ & $i$ & $S$ & 677 & $<0.001$ & 9 & 15409 \\
\hline$i$ & $i$ & $\mathrm{Clu}$ & 689 & $<0.001$ & 9 & 15421 \\
\hline$i$ & $i$ & $\mathrm{LC}$ & 728 & $<0.001$ & 9 & 15460 \\
\hline$i$ & $i$ & $i$ & 731 & $<0.001$ & 6 & 15469 \\
\hline $\mathrm{LC}$ & $i$ & $i$ & 733 & $<0.001$ & 8 & 15467 \\
\hline Clu & $i$ & $i$ & 733 & $<0.001$ & 8 & 15467 \\
\hline$R$ & $i$ & $i$ & 733 & $<0.001$ & 9 & 15467 \\
\hline$S$ & $i$ & $i$ & 734 & $<0.001$ & 9 & 15468 \\
\hline $\mathrm{Clu} \times \mathrm{LC}$ & $i$ & $i$ & 734 & $<0.001$ & 10 & 15468 \\
\hline$i$ & $i$ & $O$ & & & 76 & $\mathrm{NC}$ \\
\hline
\end{tabular}

Notes: Multistate models where $\psi^{1,2}$ and $\psi^{0,2}$ are the probabilities of transition from, respectively, state 0 (absence) and state 1 (low-density) to state 2 (gregarious); $p^{0,1}$ is the probability of misclassifying a cell as having an absence of locusts $(0)$ when the true state is having a low-density population (1). Models are compared with $\triangle \mathrm{AIC}$ (the change in the Akaike information criterion due to the model) and Akaike weight $(w) ; k$ indicates the number of parameters of the model, + refers to additive effects, $\times$ to interactions, and NC to models that did not converge. Covariates used in different models are detailed in Table 1.

Wright (1986) also suggested that gregarious populations could cover distance up to $1500 \mathrm{~km}$ in a few nights. To test this regional migration hypothesis, the regional neighborhood was defined by including all cells of the study area. Autologistic modeling of the probability of state transition (from $R=0$ or 1 ) to a gregarious population $(R=2)$ is then modeled as

$$
\psi_{i, t}^{R, 2}=\frac{\exp \left(\beta_{0, t}+\beta_{1, t} \hat{\bar{\pi}}_{t}^{n_{i}}\right)}{1+\exp \left(\beta_{0, t}+\beta_{1, t} \hat{\bar{\pi}}_{t}^{n_{i}}\right)} .
$$

Multistate and autologistic models can be implemented in a Bayesian framework or in a maximum likelihood framework using the program PRESENCE (available online). ${ }^{12}$ We compared more than 25 models, some with up to 40 parameters. Due to the autologistic component of the model, we used maximum likelihood for computational performance and time. In a Bayesian framework, one is required to calculate the number of adjacent cells in the gregarious population state for each cell at each occasion and for each simulation. However, PRESENCE can only estimate the $\beta$ parameters of autologistic models restricted to two states. Therefore, we used a two steps procedure in which we first ran different multistate models in order to (1) estimate the site transition between the absence of locust and gregarious populations $\left(\hat{\psi}^{0,2}\right)$, (2) estimate the rate of detection of scattered populations $\left(\hat{p}^{0,1}\right)$, and (3) perform model selection among the different environmental predictors. Then, since $\hat{p}^{0,1}$ was low (see Results),

12 http://www.mbr-pwrc.usgs.gov/software/presence.html meaning that most sites with no locust observed were likely to be sites with locusts not detected, we pooled the initial states of absence and low abundance into a single state, retained gregarious populations as the second state, and estimated autologistic parameters via maximum likelihood using PRESENCE. We reestimated parameters from the best model with environmental covariates in order to test for the sensitivity of the estimates to the pooling of the two first states and to perform model selection between autologistic parameters and environmental factors.

Model selection was based on Akaike's information criterion (AIC; Akaike 1974). We also used AIC weight (w) as a measure of relative support for each model ( $w$ ranges from 0 to 1 , with 1 indicating maximum support; Burnham and Anderson 2002).

\section{Results}

\section{Modeling}

The probability of detecting a population at low density varied over a year, with an apparent declining trend (see Appendix), but was low on average $\left(\hat{p}_{\text {max }}^{0,1}=\right.$ $0.284 \pm 0.018[$ mean $\pm \mathrm{SE}], \hat{p}_{\min }^{0,1}=0.041 \pm 0.012, \bar{p}^{0,1}=$ $0.162 \pm 0.035)$. Therefore, even if a cell had a few individuals present, they were unlikely to be detected. The resultant probability of transition from absence to outbreak populations was very low $\left(\psi^{0,2}<0.0001\right)$, and there was no effect of any spatial or temporal covariate on this probability of transition (Tables 1 and 2).

On the other hand, the transition probability from low-density to outbreaking populations varied in time and space (Table 2). We found a seasonal effect, with 

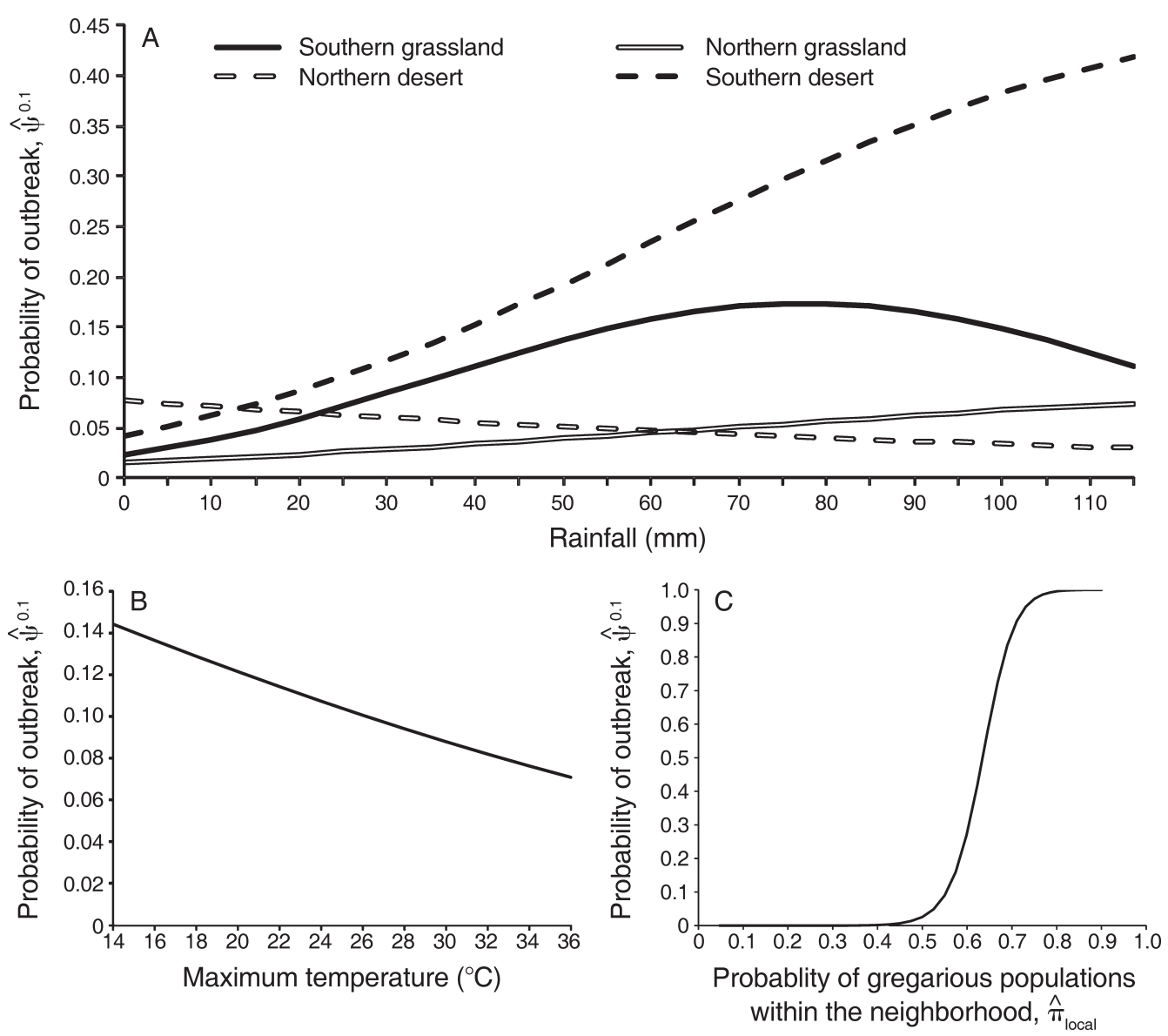

FIG. 3. Parameter estimates of (A) the probability of outbreak $\left(\psi^{0,1}\right)$ for a given rainfall (mean rainfall per season per grid cell) in the four different areas defined by the cluster analysis (southern vs. northern cells) and the presence or absence of vegetation; (B) the probability of outbreak $\left(\psi^{0,1}\right)$ for a given temperature (mean maximum temperature per season per grid cell) in the southern vegetation cluster; (C) the probability of outbreak $\left(\psi^{0,1}\right)$ with the effect of local neighbor sites. Parameters estimates come from the model with the highest AIC weight among a set of models including an autologistic component and/or environmental covariates. The $x$-axis shows the mean probability of a site with a gregarious population within a neighborhood of $n_{i}$ sites $\left(\hat{\pi}_{\text {local }}\right)$ at the previous time step.

higher probability of outbreak in summer $\left(\hat{\psi}_{\text {summer }}^{1,2}=\right.$ 0.092 ; CI, $0.071-0.118$ vs. $\hat{\psi}_{\text {spring }}^{1,2}=0.068$; CI, $0.053-$ 0.888 and $\hat{\psi}_{\text {autumn }}^{1,2}=0.067$; CI, 0.051-0.086). Gregarious populations were overall more likely to occur in the southern cluster than in the northern cluster, and more likely in the desert than in grassland areas of the northern cluster $\left(\hat{\psi}_{\text {Sveg }}^{1,2}=0.097 ; \mathrm{CI}, 0.075-0.124 ; \hat{\psi}_{\mathrm{Sd}}^{1,2}=\right.$ 0.095 ; CI, 0.073-0.123; $\hat{\psi}_{\text {Nveg }}^{1,2}=0.037$; CI, 0.026-0.051; $\hat{\psi}_{\mathrm{Nd}}^{1,2}=0.062$; CI, 0.041-0.093; where $\mathrm{S}$ is southern, $\mathrm{N}$ is northern, veg is vegetation and $\mathrm{d}$ is desert).

The amount of rainfall per season explained a significant amount of variation in outbreaks. The model with an interaction between rainfall and the four areas defined above outperformed all models, including models describing spatial variation by latitude and longitude coordinates of cells and temporal variation by a seasonal effect (Table 2). In the most supported model, each area responded differently to rainfall; the northern regions show little variation in likelihood of outbreaks with variation in rainfall, whereas outbreaks in the southern regions are sensitive to amounts of rainfall (Fig. 3A). With increasing rainfall in the southern areas, gregarious populations have a greater probability of emerging in the desert than in grassland areas (Fig. 3A). Since temperature data were lacking in the desert, we only modeled the effect of temperature in cells belonging to the southern and northern clusters and in cells with vegetation. We found no significant effect of temperature in the northern cluster and a negative effect in the southern cluster (Fig. 3B), with outbreaks being less likely to occur with increasing maximum temperature.

Between the two competing models with an autologistic structure, the model considering only a local neighborhood effect had the lowest AIC; however, it did not outperform the model with environmental covariates. Overall, the best model included all covariates, indicating that both environmental factors and local 
dispersal explained outbreaks (Table 3). As hypothesized, the $\hat{\beta}$ associated with the autologistic part of the model was positive $(\hat{\beta}=6.357$; CI, 5.641-7.073), indicating that a site will have a higher probability of an outbreak if the local probability of having a gregarious population is high (Fig. 3C).

\section{Discussion}

We show that the application of multistate occupancy models to locust data is a useful tool for explicitly quantifying temporal and spatial patterns of outbreak events, allowing testing of competing hypotheses about environmental and demographic processes. By accounting for the low rates of locust detection, we found that an outbreak transition is unlikely to occur in the absence of an existing scattered population of locusts. In cells with a scattered population, variation in outbreak probability was better explained by the climatic clusters than by vegetation type, suggesting a stronger impact of the climatic regime on outbreak probabilities. Indeed, the response curve of outbreak events to rainfall shows different patterns between the northern cluster, which is under a tropical regime, and the southern cluster, which is under a temperate regime. On average, outbreak events were less likely to happen in the north than in the south, and less likely in grassland areas than in desert areas. In the north, amounts of rainfall did not explain much variation in outbreaks events. In contrast, rainfall was positively correlated to probabilities of outbreaks in the southern cluster, with a faster response in the desert than in grassland areas. Given the sensitivity of locust population growth to large rainfall events and the assumed positive effects of rainfall on both the availability of food and conditions for egg laying and development (Popov et al. 1991, Hunter et al. 2001, Sword et al. 2010, Deveson 2013), a higher probability of outbreak under tropical conditions and in grassland areas rather than under temperate conditions or in desert areas could have been expected. The weak sensitivity to rainfall in the northeast part of eastern Australia indicates that rain is not a limiting factor in this area, probably due to a fairly constant level of humidity year-round, especially in the herbaceous area, which is more represented in our tropical cluster than the desert area (see Fig. 1B and C). Rain might even have a negative effect on egg laying and development in the North by inducing levels of moisture in the soil that are too high, and especially by promoting pathogen persistence and spread (Launois 1978, Chapman et al. 1986, Farrow 1991). Moreover, the higher probability of outbreak in desert than in grassland areas could be due to the patchy distribution of ephemeral vegetation growing rapidly after rainfall events in the desert (Letnic and Dickman 2005). Spatially limited vegetation patches will concentrate locusts on the same spots. The subsequent increase in local densities is likely to rapidly induce a phase change from solitarious to gregarious
TABLE 3. Single-state model selection results for the Australian locust data set.

\begin{tabular}{lrrrc}
\hline \hline \multicolumn{1}{c}{$\psi^{0,1}$} & $\Delta$ AIC & \multicolumn{1}{c}{$w$} & $k$ & Deviance \\
\hline Clu $\times$ LC $\times R+T+\hat{\pi}_{\text {local }}$ & 0 & $<0.999$ & 18 & 3481 \\
Clu $\times$ LC $\times R+T+\hat{\pi}_{\text {regional }}$ & 42 & $<0.001$ & 18 & 3523 \\
Clu $\times$ LC $\times R+T$ & 170 & $<0.001$ & 17 & 3655 \\
$\hat{\pi}_{\text {local }}$ & 193 & $<0.001$ & 5 & 3726 \\
$\hat{\pi}_{\text {regional }}$ & 289 & $<0.001$ & 5 & 3822 \\
$i$ & 361 & $<0.001$ & 4 & 3898 \\
\hline
\end{tabular}

Notes: Single-state models where $\psi^{0,1}$ is the probability of transition from state 0 (absence or low-density) to state 1 (gregarious). The probability of misclassification is assumed to be 0 . Covariates used in different models are detailed in Table 1. See Table 2 for explanations of model selection parameters.

populations, leading to outbreaks and mass migration (Sword et al. 2010).

Concerning the influence of temperature on outbreaks, we found a negative impact of average maximum temperature on outbreaks in areas of the Southern cluster with vegetation, but the scarcity of a spatial series of temperature data does not allow inferences to be drawn in other regions. However, the similarity between the clusters of rain and the clusters of temperature indicates that, on average, higher temperatures are associated with stronger precipitation in the northern part of Australia and are less likely to induce outbreak events. Above all, the different responses of locusts to climatic factors emphasize the key role of spatial environmental heterogeneity and the need to account for it when analyzing population fluctuations (Bjørnstad and Grenfell 2001).

Another specificity of our modeling approach is the addition of an autologistic component as a proxy for the migration process. It allowed us to discriminate between the two competing hypotheses of local vs. regional migration of gregarious populations. Overall, we show that even if environmental predictors explain more of the large-scale variation in outbreak events than migration, both factors seem to play a role in the occurrence of outbreaks. Under favorable climatic conditions, locust density increases until reaching a threshold density that causes the gregarization of individuals. Our results support the hypothesis of a local propagation of these gregarious populations as opposed to the hypothesis of a local outbreak event that would diffuse over a long distance (Farrow 1991). This finding is also consistent with a recent study based on microsatellite data and computer simulations, which revealed that despite the apparent genetic homogeneity of the APL at the continental scale, the rate of dispersal could be several orders of magnitude lower than the $10 \%$ typically considered as required for the demographic connectivity of populations (Chapuis et al. 2011).

From a management point of view, our results emphasize the need to account for spatiotemporal variation in weather factors, especially rainfall, and green vegetation in order to develop a forecasting model for locust outbreaks and inform locust control strate- 
gies. Such integration has been attempted by the APLC, using satellite vegetation greenness, weather and atmospheric circulation data, as well as locust surveillance data, to assist locust forecasting and population control decisions (Bryceson et al. 1993, Hamilton and Bryceson 1993, Deveson and Hunter 2002). Locust migration still remains as a key biological process to incorporate into the forecasting and management framework for the APL. Conclusions from recent studies on locust migration in Australia are that demographic flow is driven by weather systems that produce winds in a variety of directions and lead to locust migration across much of the geographical range of the species (Deveson et al. 2005, Deveson and Walker 2005). In the present study, the autologistic model indicates that locust migration acts as a local rather than a regional contagious process. Gregarious populations are more likely to propagate through migration over distances $<400 \mathrm{~km}$. At wider regional scales, weather conditions and land cover remain the main factors influencing outbreak probabilities. Our results suggest that locust population forecasting could be improved by implementing both heterogeneity in environmental features and autocorrelation in the dispersal process in hierarchical spatial models as is being increasingly applied to predict disease spread in epidemiology (e.g., Wimberly et al. 2008, Wang et al. 2010, Adegboye and Koze 2012). Achieving better predictions of locust outbreak occurrence will enable early population control measures and contribute to more effective preventive management.

In this study, we restricted our analyses of APL dynamics to the emergence of outbreaks. We therefore focused on specifically modeling the probability of transitions from scattered to gregarious populations. However, other transitions are also of ecological and/or management interest and could be modeled upon availability of field data and potential covariates. For example, it would be useful to model population transition to a low density after an outbreak event. However, multistate models consist of several multinomial distributions in which the sum of probabilities equals 1 . Therefore, each probability of the multinomial cannot be modeled independently as a function of a covariate. Modeling several probabilities from the same multinomial as a function of covariates requires reparameterizing the model using different methods such as cumulative probabilities, continuation-ratios, or adjacent categories (see McCullagh and Nelder 1989).

Importantly, our framework can be seen as a step towards bridging the gap between mechanistic modeling and more phenomenological models in the spatial analysis of fluctuating populations. Multistate, multiseason, occupancy models belong to the class of hierarchical models or state-space models, where the variability of the state of the population (here classes of density) is separated between the process variability that is of ecological interest and the observation error (in our case, the probability of misclassifying classes of abun- dance due to imperfect detection [Clark and Bjørnstad 2004, Royle and Dorazio 2008]). Not only do hierarchical models allow estimation of the biological variability of the system, they enable the development of more mechanistic models by fitting population dynamics models to time-series data (de Valpine 2002, Schurr et al. 2012). In our model, the transition from low-density to gregarious populations corresponds to the underlying behavioral process of gregarization. A next step could consist of including individual demographic traits such as survival and fecundity of solitarious and gregarious individuals, which are traits that can vary in locusts in a density-dependent manner (Pener and Simpson 2009). Building a more complex population model such as this, for example, would allow one to test which parameters in the population model respond to environmental variation, and determine the shape of its response curve or the importance of group/individual heterogeneity influences. Given the combination of global warming, habitat change, and increasing risks of biological invasions, more mechanistic models are necessary to more effectively predict population dynamics in our changing world (Benton et al. 2006, Schurr et al. 2012).

\section{ACKNOWLEDGMENTS}

We are grateful to M-P. Chapuis and X. Lambin for useful comments on this work. This research was funded by an Australian Research Council Linkage grant (LP0669080) on Australian plague locust population genetics and migratory behavior.

\section{Literature Cited}

Adegboye, O. A., and D. Koze. 2012. Disease mapping of Leishmaniasis outbreak in Afghanistan: spatial hierarchical Bayesian analysis. Asian Pacific Journal of Tropical Disease 2(4):253-259.

Akaike, H. 1974. A new look at the statistical model identification. IEEE Transactions on Automatic Control 19:716-723.

Augustin, N. H., M. A. Mugglestone, and S. T. Buckland. 1996. An autologistic model for the spatial distribution of wildlife. Journal of Applied Ecology 33:339-347.

Babah, M. A. O., and G. A. Sword. 2004. Linking locust gregarization to local resource distribution patterns across a large spatial scale. Environmental Entomology 33:15771583.

Baltensweiler, W., and A. Fischlin. 1988. The larch budmoth in the Alps. Pages 331-351 in A. Berryman, editor. Dynamics of forest insect populations: patterns, causes, implications. Plenum Press, New York, New York, USA.

Benton, T. G., S. J. Plaistow, and T. N. Coulson. 2006. Complex population dynamics and complex causation: devils, details and demography. Proceedings of the Royal Society B 273:1173-1181.

Bjørnstad, O. N., and B. T. Grenfell. 2001. Noisy clockwork: time series analysis of population fluctuations in animals. Science 293:638-643.

Bjørnstad, O. N., R. A. Ims, and X. Lambin. 1999. Spatial population dynamics: analyzing patterns and processes of population synchrony. Trends in Ecology and Evolution 14: 427-432.

Bled, F., J. A. Royle, and E. Cam. 2010. Hierarchical modeling of an invasive spread: the Eurasian Collared-Dove Streptopelia decaocto in the United States. Ecological Applications 21:290-302. 
Bryant, E. 1985. The southern oscillation and climatic effects in Australia. Wollongong Studies in Geography 16. University of Wollongong, Wollongong, New South Wales, Australia.

Bryceson, K. P. 1991. Likely locust infestation areas in Western New South Wales, Australia, located by satellite. Geocarto International 6.4:21-37.

Bryceson, K. P., D. M. Hunter, and J. G. Hamilton. 1993. Use of remotely sensed data in the Australian Plague Locust Commission. Pages 435-439 in S. A. Corey, D. J. Dall, and W. M. Milne, editors. Pest control and sustainable agriculture. CSIRO, Melbourne, Australia.

Bryceson, K. P., and D. E. Wright. 1986. An analysis of the 1984 locust plague in Australia using multitemporal landsat multispectral data and a simulation model of locust development. Agriculture, Ecosystems and Environment 16: 87-102.

Burnham, K. P., and D. R. Anderson. 2002. Model selection and multi-model inference: a practical information-theoretic approach. Springer, New York, New York, USA.

Chapman, R. F., W. W. Page, and A. R. McCaffery. 1986. Bionomics of the variegated grasshopper (Zonocerus Variegatus) in West and Central Africa. Annual Review of Entomology 31:479-505.

Chapuis, M.-P., J.-A. M. Popple, K. Berthier, S. J. Simpson, E. Deveson, P. Spurgin, M. J. Steinbauer, and G. A. Sword. 2011. Challenges to assessing connectivity between massive populations of the Australian plague locust. Proceedings of the Royal Society B 278:3152-3160.

Clark, J. S., and O. N. Bjørnstad. 2004. Population time series: process variability, observation errors, missing values, lags, and hidden states. Ecology 85:3140-3150.

de Valpine, P. 2002. Review of methods for fitting time-series models with process and observation error and likelihood calculations for nonlinear, non-Gaussian state-space models. Bulletin of Marine Science 70:455-471.

Despland, E., J. Rosenberg, and S. J. Simpson. 2004. Landscape structure and locust swarming: a satellite's eye view. Ecography 27:381-391.

Deveson, E. D. 2013. Satellite normalized difference vegetation index data used in managing Australian plague locusts. Journal of Applied Remote Sensing 7(1):075096.

Deveson, E. D., V. A. Drake, D. M. Hunter, P. W. Walker, and H. K. Wang. 2005. Evidence from traditional and new technologies for northward migrations of Australian plague locusts (Chortoicetes terminifera) (Walker) (Orthoptera: Acrididae) to western Queensland. Austral Ecology 30:920935.

Deveson, E. D., and P. Walker. 2005. Not a one-way trip: historical distribution data for Australian plague locusts support frequent seasonal exchange migrations. Journal of Orthoptera Research 14(1):91-105.

Deveson, T., and D. M. Hunter. 2002. The operation of a GISbased decision support system for Australian locust management. Insect Science 9(4):1-12.

Dormann, F. C., et al. 2007. Methods to account for spatial autocorrelation in the analysis of species distributional data: a review. Ecography 30:609-628.

Drake, V. A., and R. A. Farrow. 1983. The nocturnal migration of the Australian plague locust, Chortoicetes terminifera (Walker) (Orthoptera: Acrididae): quantitative radar observations of a series of northward flights. Bulletin of Entomological Research 73:567-585.

Farrow, R. A. 1990. Flight and migration in acridoids. Pages 227-314 in R. F. Chapman and A. Joern, editors. Biology of grasshoppers. Wiley, New York, New York, USA.

Farrow, R. A. 1991. Implications of potential global warming on agricultural pests in Australia. EPPO Bulletin 21:683-696.

Goswami, V. R., L. L. Getz, J. A. Hostetler, A. Ozgul, and M. K. Oli. 2011. Synergistic influences of phase, density, and climatic variation on the dynamics of fluctuating populations. Ecology 92:1680-1690.
Gray, L. J., G. A. Sword, M. L. Anstey, F. J. Clissold, and S. J. Simpson. 2009. Behavioural phase polyphenism in the Australian plague locust (Chortoicetes terminifera). Biology Letters 5:306-309.

Hamilton, J. G., and K. P. Bryceson. 1993. Use of enhanced GMS weather satellite data in locust forecasting. Pages 444448 in S. A. Corey, D. J. Dall, and W. M. Milne, editors. Pest control and sustainable agriculture. CSIRO, Melbourne, Australia.

Hendon, H. H., D. W. J. Thompson, and M. C. Wheeler. 2007. Australian rainfall and surface temperature variations associated with the southern hemisphere annular mode. Journal of Climate 20:2452-2467.

Hunter, D. M., P. W. Walker, and R. J. Elder. 2001. Adaptations of locusts and grasshoppers to the low and variable rainfall of Australia. Journal of Orthoptera Research 10:347-351.

Johnson, D. M., O. N. Bjørnstad, and M. Liebhold. 2004. Landscape geometry and travelling waves in the larch budmoth. Ecology Letters 7:967-974.

Johnson, D. M., O. N. Bjørnstad, and M. Liebhold. 2006. Landscape mosaic induces travelling waves of insect outbreaks. Oecologia 148:51-60.

Knape, J., and P. de Valpine. 2011. Effects of weather and climate on the dynamics of animal population time series. Proceedings of the Royal Society B 278:985-992.

Kvasnes, M. A. J., T. Storaas, H. C. Pedersen, S. Bjørk, and E. B. Nilsen. 2010. Spatial dynamics of Norwegian tetraonid populations. Ecological Research 25:367-374.

Launois, M. 1978. Modélisation écologique et simulation opérationnelle en acridologie: Application à Oedaleus senegalensis (Krauss, 1877). Ministère de la Coopération, Paris, France.

Letnic, M., and C. R. Dickman. 2005. The responses of small mammals to patches regenerating after fire and rainfall in the Simpson Desert, central Australia. Austral Ecology 30:2439.

Liebhold, A., W. D. Koenig, and O. N. Bjørnstad. 2004. Spatial synchrony in population dynamics. Annual Review of Ecology, Evolution, and Systematics 35:467-490.

Liebhold, A. M., R. E. Rossi, and W. P. Kemp. 1993. Geostatistics and geographic information systems in applied insect ecology. Annual Review of Entomology 38:303-327.

MacKenzie, D. I. 2006. Occupancy estimation and modeling: inferring patterns and dynamics of species occurrence. Academic Press, New York, New York, USA.

MacKenzie, D. I., J. D. Nichols, J. E. Hines, M. G. Knutson, and A. B. Franklin. 2003. Estimating site occupancy, colonization and local extinction when a species is detected imperfectly. Ecology 84:2200-2207.

MacKenzie, D. I., J. D. Nichols, G. B. Lachman, S. S. Droege, J. A. Royle, and C. A. Langtimm. 2002. Estimating site occupancy rates when detection probabilities are less than one. Ecology 83:2248-2255.

MacKenzie, D. I., J. D. Nichols, M. E. Seamans, and R. J. Gutiérrez. 2009. Modeling species occurrence dynamics with multiple states and imperfect detection. Ecology 90:823-835.

Martin, J., C. L. McIntyre, J. E. Hines, J. D. Nichols, J. A. Schmutz, and M. C. MacCluskie. 2009. Dynamic multistate site occupancy models to evaluate hypotheses relevant to conservation of Golden Eagles in Denali National Park, Alaska. Biological Conservation 142:2726-2731.

McCullagh, P., and J. A. Nelder. 1989. Generalized linear models. Second edition. Chapman and Hall, London, England.

Miller, D. A., J. D. Nichols, B. T. McClintock, E. H. C. Grant, L. L. Bailey, and L. Weir. 2011. Improving occupancy estimation when two types of observational error occur: nondetection and species misidentification. Ecology 92:14221428. 
Nichols, J. D., J. E. Hines, D. I. Mackenzie, M. E. Seamans, and R. J. Gutierrez. 2007. Occupancy estimation and modeling with multiple states and state uncertainty. Ecology 88:1395-1400.

Pener, M. P., and S. J. Simpson. 2009. Locust phase polyphenism: an update. Pages 1-272 in S. J. Simpson and M. P. Pener, editors. Advances in insect physiology. Academic Press, New York, New York, USA.

Popov, G. B., J. F. Duranton, and J. Gigault. 1991. Etude ecologique des biotopes du criquet pelerin Schistocerca gregaria (Forskal, 1775) en Afrique Nord-Occidentale: mise en evidence et description des unites territoriales ecologiquement homogenes. CIRAD-PRIFAS, Montpellier, France.

Rossi, R. E., D. J. Mulla, A. G. Journel, and E. H. Franz. 1992. Geostatistical tools for modelling and interpreting ecological spatial dependence. Ecological Monographs 62:277-314.

Royle, J. A., and R. M. Dorazio. 2008. Hierarchical modeling and inference in ecology: the analysis of data from populations, metapopulations and communities. First edition. Academic Press, San Diego, California, USA.

Royle, J. A., and W. A. Link. 2006. Generalized site occupancy models allowing for false positive and false negative errors. Ecology 87:835-841.

Schurr, F. M., et al. 2012. How to understand species' niches and range dynamics: a demographic research agenda for biogeography. Journal of Biogeography 39:2146-2162.

Simpson, S. J., A. R. McCaffery, and B. F. Hägele. 1999. A behavioural analysis of phase change in the desert locust. Biological Reviews 74:461-480.

Singleton, G. R., S. Belmain, P. R. Brown, K. Aplin, and N. M. Htwe. 2010. Impacts of rodent outbreaks on food security in Asia. Wildlife Research 37:355-359.

Stenseth, N. C., et al. 1998. From patterns to processes: phase and density dependencies in the Canadian lynx cycle. Proceedings of the National Academy of Sciences USA 95: $15430-15435$.

Stige, L. C., K.-S. Chan, Z. Zhang, D. Frank, and N. C. Stenseth. 2007. Thousand-year-long Chinese time series reveals climatic forcing of decadal locust dynamics. Proceedings of the National Academy of Sciences USA 104:1618816193.

Sword, G. A., M. Lecoq, and S. J. Simpson. 2010. Phase polyphenism and preventative locust management. Journal of Insect Physiology 56:949-957.
Symmons, P. M., and D. E. Wright. 1981. The origins and course of the 1979 plague of the Australian plague locust, Chortoicetes terminifera (Walker) (Orthoptera: Acrididae), including the effect of chemical control. Acrida 10:159-190.

Tian, H., L. C. Stige, B. Cazelles, K. L. Kausrud, R. Svarverud, N. C. Stenseth, and Z. Zhang. 2011. Reconstruction of a 1,910-y-long locust series reveals consistent associations with climate fluctuations in China. Proceedings of the National Academy of Sciences USA 108:14521-14526.

Veran, S., K. J. Kleiner, R. Choquet, J. A. Collazo, and J. D. Nichols. 2012. Modeling habitat dynamics accounting for possible misclassification. Landscape Ecology 27:943-956.

Wang, G., R. B. Minnis, J. L. Belant, and C. L. Wax. 2010. Dry weather induces outbreaks of human West Nile virus infections. BioMed Central Infectious Diseases 10:38.

Ward, J. H. 1963. Hierarchical grouping to optimize an objective function. Journal of the American Statistical Association 58:236-244.

Wardhaugh, K. G. 1986. Diapause strategies in the Australian plague locust (Chortoicetes terminifera Walker). Pages 89104 in F. Taylor and R. Karban, editors. The evolution of insect life cycles. Springer-Verlag, New York, New York, USA.

Wimberly, M. C., A. D. Baer, and M. J. Yabsley. 2008. Enhanced spatial models for predicting the geographic distribution of tick-borne pathogens. International Journal of Health Geographics 7:15.

Wright, D. E. 1986. Economic assessment of actual and potential damage to crops caused by the 1984 locust plague in south-eastern Australia. Journal of Environmental Management 23:293-308.

Wright, D. E. 1987. Analysis of the development of major plagues of the Australian plague locust, Chortoicetes terminifera (Walker) using a simulation model. Austral Journal Ecology 12:423-437.

Wright, D. E., and P. M. Symmons. 1987. The development and control of the 1984 plague of the Australian plague locust, Chortoicetes terminifera (Walker). Crop Protection 6: 13-19.

Yackulic, C. B., J. Reid, R. Davis, J. E. Hines, J. D. Nichols, and E. Forsman. 2012. Neighborhood and habitat effects on vital rates: expansion of the Barred Owl in the Oregon Coast Ranges. Ecology 93:1953-1966.

\section{Supplemental Material}

\section{Ecological Archives}

The Appendix is available online: http://dx.doi.org/10.1890/14-0183.1.sm 